\section{THREE CASES OF PSITTACOSIS WITH TWO DEATHS}

\author{
BY \\ H. R. FISHER, M.B., Ch.B.Liverp. \\ AND \\ R. J. HELSBY, M.C., L.R.C.P., L.R.C.S.ED.
}

It has been generally accepted that psittacosis is usually caused by contact with parrots, and it must be admitted that the human cases of the disease that were being so regularly reported in the medical journals during the early part of 1930 appeared to cease after the regulations of the Ministry of Health prohibiting the importation of parrots came into force in May of that year. The small outbreak described below suggests, however, that psittacosis may still be occurring unrecognized, and that the possible contact with birds of patients suffering from symptoms suggestive of an atypical pneumonia or enteric fever should not be overlooked, especially as these three cases show the very real danger of human case-to-case infection in psittacosis.

We were fortunate in obtaining the collaboration of the Ministry of Health in the investigation of these cases, and also of Dr. Dorothy S. Russell and Dr. S. P. Bedson of the London Hospital, both of whom have kindly contributed notes on the pathological and bacteriological findings, which appear below; and we wish to record our appreciation of the assistance given to us.

On October 21st, 1930, one of us (H. R. F.) returned from a holiday and found that his locumtenent had been greatly puzzled by a case which eventually appeared to have been one of pneumonia.

\section{CASE I}

The patient, a lady, aged 45, on a visit with another lady, began to feel unwell on October 11th, the day after her arrival. During the first four days of her illness, the only symptoms were severe headache, pyrexia $\left(103^{\circ} \mathrm{F}\right.$.), and quickened respirations; later she had constipation, followed, after a dose of Epsom salts, by a somewhat severe diarrhoea. She also had attacks of epistaxis both in the early days and later in her illness. After the fourth day signs of pneumonia developed at the angle of the right scapula; there was little cough, no sputum, and no dyspnoea. This condition persisted until October 19th, when the temperature began to fall. By October 21 st the temperature was normal and the patient felt pretty well. The signs of consolidation were quite marked in the region mentioned, and there were coarse crepitations. The temperature rose to $100^{\circ}$ on the following evening, but came down in a few hours, and remained normal throughout her convalescence. The signs at the angle of the right scapula remained practically unchanged until October 28th, when they suddenly disappeared in one night. Her friends noticed that, during the acute period of her illness, the patient showed slowness and thickness of speech, though this was quite foreign to her usual condition. During convalescence her speech became normal, but she was able to remember very little about her illness.

\section{CASE II.}

Towards evening on October 26th the second lady, aged 35, who had slept in the same bed as the first patient, and had nursed her during the first week of her illness, felt unwell and shivery. On the following morning she was very drowsy, and suffered acutely from occipital headache and photophobia; her temperature was $102^{\circ}$, pulse 110 , respirations 30 . On October 28th her temperature rose to $104^{\circ}$; the headache and photophobia were still severe, and the patient said she felt as if her hair was being pulled. There were no physical signs, no cough, sputum, or dyspnoea. There was no tenderness anywhere except in the occipital region. The bowels were acting normally, and, except for some abdominal distension, she had no gastro-intestinal symptoms. On October 29th the temperature rose to $104.5^{\circ}$, the pulse was 116 , and the respirations 30 ; the patient had some epistaxis, but her condition was much the same, except that the drowsiness and photophobia were less marked.

In the meantime it had been discovered, in the course of a chance conversation with the convalescent patient, that the ladies had an aviary at their home, and that the first patient had nursed a sick budgerigar for some days immediately preceding her illness. These facts, coupled with the suggestive course of the illness, prompted communication with the Ministry of Health.

On October 30th the temperature rose to $105^{\circ}$, the pulse was 118 , respirations 30 ; there was slight impairment of note and diminution of the breath sounds at the angle of the right scapula. These signs became more pronounced during that day. On October 31st the temperature remained at $105^{\circ}$ (there had been no remissions), respirations were 32 , and the pulse varied between 102 and 120 . The signs in the chest had become marked; there was bronchial breathing, dullness in the area already mentioned, and rales all over. Cough was slight, and there was no dyspnoea. The patient vomited a little, and started menstruating prematurely. S.U.P. 36 was given on this day with no result. This patient also showed some slowness of cerebration, and " wandered" during the night, though, when she finally understood a question, she answered intelligently but in a slow, hesitant manner. On November 1st the temperature was $104.5^{\circ}$, pulse 110 , respirations 38 ; her condition was much the same. On November 2nd the temperature was $100^{\circ}$ in the morning, with pulse 96 , respirations 32 ; in the evening it was up again to $104^{\circ}$. On November 3 rd and 4th the patient was worse; she took no interest in her surroundings, and moaned and sighed loudly for long periods; the first patient had also moaned a great deal. Her temperature was swinging between $99^{\circ}$ and $104^{\circ}$. At midnight (November 5th-6th) the patient became collapsed; but the pulse soon strengthened and the temperature, pulse, and respirations began to fall until at 6 a.m. they were normal. She felt much better, and chatted in her usual way. That evening, however, the temperature rose to $102^{\circ}$, the pulse and respirations quickened, and she vomited. On November 7 th the temperature was swinging between $99^{\circ}$ and $104.5^{\circ}$, while the vomiting continued. On November 8th the patient became cyanosed, complained a great deal of epigastric pain, and died at midday.

It seems likely that this lady was infected by the previous patient, with whom she was in close contact during the first week of the latter's illness. Still, the possibility that she also came into contact with a sick bird before leaving home on October 10th cannot be entirely disproved ; though, as she left home on October 10th and did not begin to be ill until October 26th; the incubation period was sixteen or more days. While this is not unknown, it is less usual than one of eight to fourteen days, a period coinciding with the time of her contact with an acutely ill patient.

\section{CASE III}

During the night of November 19th-20th, eleven days after the previous patient's death, a trained nurse, aged 63, who had been in attendance on both these cases, felt unwell. On November 20th she had epistaxis, and complained of frontal headache and vomiting. The pulse was 80, temperature $99.5^{\circ}$. On November 21 st she was removed to hospital. This patient was undoubtedly infected by the patients she had been nursing; at no time had she been in contact with sick birds. . During the first day after her admission into hospital there were no physical signs in the chest, but she vomited and was constipated; her temperature was $104^{\circ} \mathrm{F}$., pulse 92, 
respirations 30 . On November 23rd there was dullness over the left lower lobe, with rales over the right side. The sputum was scanty and rusty. She complained a good deal of frontal headache, and had difficulty in swallowing. After November 24th she began to have incontinence of urine and faeces, which continued until her death. On November 26th there appeared to be paralysis of the left side of the body and the right side of the face, but this passed off in a couple of days. Gradually, however, nervous symptoms became more marked, together with abdominal tumescence, drowsiness, and symptoms generally reminiscent of typhoid fever. In fact, during the last five days of her life, the patient was in a typhoid state, more or less unconscious, and with a subnormal temperature. She died on the morning of December 3rd.

Two other nurses in attendance on the first two cases remained well, and there were no other cases in the home of the original patients.

\section{SUMMARY}

The sequence of events in these cases was as follows:

1. At the end of September or the beginning of October, 1930, patient No. 1 nursed a sick budgerigar.

2. October 11 th, patient No. 1 fell ill when away from home on a visit. She eventually recovered, but as late as January 15th, 1931, was reported still to be suffering from insomnia, lack of energy, and occasional depression.

3. October 26th, patient No. 2 fell ill. She had nursed patient No. 1, had shared her bed, and was probably infected by her.

4. November 8 th, patient. No. 2 died.

5. November 19 th, patient No. 3 fell ill. She was a hospital nurse in attendance on the previous patients from October 23rd, and was infected by them.

6. December 3rd, patient No. 3 died.

The source of infection of the budgerigar was not definitely traced. A number of other birds, budgerigars, foreign finches, and a blue and white canary, had died in the aviary from an unknown cause during September and the beginning of October, and the dealer who had supplied the birds admitted to deaths among the birds in his shop. Later on this dealer was found to have been importing budgerigars from the Continent, but he maintained that the birds he had sold to these ladies were home-bred.

\section{SUMMARY OF NECROPSY (DECEMBER 4TH, 1930)}

$$
\text { BY }
$$

DOROThy S. Russell, M.D.Lond.

RESEARCH ASSISTANT, PATHOLOGiCAL INSTITUTE, LONDON HOSPITA.

Haemorrhagic broncho-pneumonia of lower lobe and of lower third of upper lobe of left lung. Early bronchopneumonia of lower lobe of right lung. Congestion and emphysema of lungs. Anthracosis, engorgement, slight subacute inflammation and sinus catarrh of bronchial lymph glands. Acute and subacute inflammation without enlargement of spleen. A few streaks of submucous haemorrhage in caecum close to ileo-caecal valve. Slight injection of mucosa throughout intestinal tract. Slight fatty degeneration of myocardium. Considerable ischaemic atrophy of kidneys. Albuminous degeneration of tubular epithelium in kidneys; fatty degeneration of a few groups of second convoluted tubules. Severe degeneration of hypertrophied media and intima in large and small arteries. Ante-mortem thrombus adherent to ulcerated intima of anterior wall of very atheromatous abdominal aorta $3 \mathrm{~cm}$. above bifurcation. Focal microscopic areas of bone formation in atheromatous and calcified intima beneath thrombus. Foramen ovale closed. Cardiovascular hypertrophy. Focal ischaemic fibrosis of myocardium. Very severe atheroma of coronary arteries. Almost complete obliteration of right coronary artery by calcareous atheroma $1 \mathrm{~cm}$. from its origin. Small area of softening and gliosis in right pulvinar. Area of gliosis and infiltration with pigment granule cells in ventral border of optic thalamus. A few petechial haemorrhages in basal ganglia. Congestion of brain." Central congestion of lobules of liver. Slight back pressure congestion of kidneys and spleen. Pitting oedema of left leg. Antemortem thrombosis of left femoral vein at brim of pelvis. Slight fatty infiltration. of parenchyma and conspicuous fatty infiltration of Kupffer cells in liver. Fine granules of lipochrome in centres of lobules of liver and in myocardium. Brown atrophy of thyroid gland. Fibrous peritoneal adhesions between omentum and loops of small intestine obliterating pouch of Douglas. Slight focal chronic perisplenitis. Slight dilatation and injection of pelvis of right kidney. Aberrant branch of right renal artery entering lower pole of right kidney. Pancreas and suprarenal bodies normal. No macroscopic abnormality in muscles of abdominal wall. No enlargement of Peyer's patches or of solitary follicles.

Macroscopic Appearances

Lungs.-There was considerable injection of the trachea and bronchi. The pleural sacs contained no free fluid. Both visceral and parietal layers of the pleura were uniformly smooth and glistening. The lungs appeared small and congested. The upper lobes were conspicuously emphysematous, while the lower lobes felt boggy and slightly crepitant. The lower lobe of the left lung was heavier and fuller than the right, and felt unevenly nodular. On section the right lung appeared very congested. The upper and middle lobes were emphysematous. In the lower lobe there were numerous small, irregular, apparently peribronchial. areas of haemorrhage ; there was no evidence of bronchitis or of consolidation ; a little blood-stained frothy fluid exuded from the cut surface on pressure, and the tissue was aerated and floated in water. The left lung, on section, appeared different. With the exception of the upper two-thirds of the upper lobe the lung looked less aerated. At the apex of the lower lobe was an area of rather firm, swollen, finely granular, dusiky, pinkish-grey consolidation. No fluid was expressed from this by gentle pressure. A similar area occupied the adjacent middle third of the upper lobe. In the remainder of the-lower lobe there were numerous, sometimes confluent, finely granular areas of pinkish-grey peribronchial consolidation lying in a haemorrhagic ground. Dirty greyish-yellow slime exuded from the centres of these areas on pressure. The intervening lung tissue was deeply congested, and on pressure exuded a little fluid and very little air.

Bronchial Lymph Glands.-These glands were small and firm. The cut surfaces were flat and grey, and mottled with deposits of soot.

Spleen.-The spleen was small and moderately elastic. The capsule over the diaphragmatic surface showed a few irregular patches of fibrous thickening, between which were numerous pin-point glistening fibrous granulations. On section the cut surfaces were flat and firm. The trabeculae were easily seen, but the Malpighian bodies were indistinguishable. The pulp was a dull pink mottled with numerous small patches of deep red. There was no macroscopic evidence, therefore, of inflammation.

\section{Microscopical Appearances}

Lungs.-Blocks from the right lower lobe, the consolidated areas in the middle third of the left upper lobe and the apex of the left lower lobe, and from the bronchopneumonic part of the left lower lobe, were taken for examination. In the right lower lobe some bronchioles were partly or completely filled with red corpuscles; most were filled with necrosed desquamated epithelium, ghosts of round cells, amorphous granular lumps, a few red corpuscles, and masses of cocci and bacilli. A few alveoli at the periphery of the section were emphrysematous or partly collapsed; the remainder were filled with albuminous coagulum and a few red corpuscles, occasionally with many red corpuscles. They contained no fibrin, but everywhere were large numbers of Gram-positive cocci and Gram-positive and Gram-negative bacilli. Of these, 